\title{
Optical Simulation Study on the Effect of Diffusing Substrate and Pillow Lenses on the Outcoupling Efficiency of Organic Light Emitting Diodes
}

\author{
Su Seong Jeong and Jae-Hyeon Ko* \\ Department of Physics, Hallym University, Chuncheon 200-702, Korea
}

(Received March 13, 2013 : revised May 22, 2013 : accepted May 27, 2013)

\begin{abstract}
The effect of diffusing substrate and pillow lenses on the outcoupling efficiency of organic light-emitting diodes (OLEDs) was studied by optical simulation based on the point-dipole model. The diffusing substrate included Mie scatterers by which the condition of total internal reflection could be broken. The finite-difference time-domain method was used to obtain the intensity distribution on the transparent electrode of an OLED, which was used as a light source to carry out a ray-tracing simulation of the OLED and the diffusing substrate. It was found that the outcoupling efficiency of the OLED was sensitive to the thickness of organic layers and could be increased by $21.0 \%$ by adopting a diffusing substrate in which Mie scatterers whose radius was $2.0 \mu \mathrm{m}$ were included at the density of $10^{7} \mathrm{~mm}^{-3}$ and by $65.5 \%$ by forming one pillow lens with the radius of $2 \mathrm{~mm}$ on the front surface of the glass substrate. This study revealed that the outcoupling efficiency could be improved by adopting diffusing substrate and pillow lenses along with the optimization of the thickness of each layer in the OLED.
\end{abstract}

Keywords: OLED, Outcoupling, Diffusing substrate, Optical Simulation

OCIS codes : (120.2040) Displays; (150.2950) Illumination; (230.3670) Light-emitting diodes

\section{INTRODUCTION}

OLEDs have recently attracted great attention due to their excellent performance in display devices and illumination devices [1,2]. Visible light is generated from the organic emitting layer, where the injected holes and electrons recombine. However, a substantial portion of the generated light is trapped in the organic layers and the glass substrate because of their high refractive indices [3]. Many attempts have been made to improve the outcoupling efficiency (OCE) of OLEDs [1-5], one of which is to apply a diffusing structure [6-9]. Using a glass substrate as a diffusing layer in OLEDs may be very cost-effective in many outcoupling designs. In our previous study [10], we reported the optical simulation results for an OLED with a scatterer-included glass substrate based on a ray-tracing technique. It was shown that the incorporation of Mie scatterers into the glass substrate increased the OCE substantially. However, the ray-tracing technique has some limitations in simulating the optical performance of OLEDs because the thicknesses of the organic layers and the transparent indium-tin-oxide (ITO) layer are on the order of $100 \mathrm{~nm}$, which is much smaller than the wavelength of visible light. In this respect, more rigorous analysis is necessary to simulate OLEDs, such as through the finitedifference time-domain (FDTD) method [11, 12]. The purpose of this study was to investigate the effect of a diffusing substrate (a glass substrate into which Mie scatterers are incorporated) on an OLED structure by combining FDTD and ray-tracing methods.

\section{SIMULATION}

Figure 1 shows a schematic structure of the OLED studied by the FDTD method to calculate the electromagnetic waves propagating through the planar structure. A commercially available software (FDTD Solutions, Lumerical Solutions, Vancouver, Canada) was used to carry out the simulation. The OLED in Fig. 1 is a bottom-emitting type consisting of a metal cathode, an electron-transporting layer (ETL), a hole-transporting layer (HTL), an ITO layer and a glass substrate. The simulation area was $10 \times 10 \mu \mathrm{m}^{2}$. The refractive

\footnotetext{
*Corresponding author: hwangko@hallym.ac.kr

Color versions of one or more of the figures in this paper are available online.
} 
indices of these layers were the same as those reported in Ref. [3]. A point-dipole radiation source at a wavelength of $550 \mathrm{~nm}$ was placed at the center of the interface between the HTL and ETL. The oscillation direction of the point dipole was set along the three orthogonal directions, two in the OLED plane and one normal to the OLED plane. The far-field angular radiant intensity distribution on the ITO layer was imported into the ray-tracing software program (ASAP, Breault Research Organization, Tucson, USA) as the emitting ray distribution of an imaginary flat light source. This imaginary light source, with an area of $0.5 \times 0.5 \mathrm{~mm}^{2}$, was located at the center of the glass substrate. A glass substrate with an area of $2 \times 2 \mathrm{~mm}^{2}$ and a thickness of $0.7 \mathrm{~mm}$ acted as the diffusing layer into which scattering particles were incorporated.

Figure 2 shows a schematic structure of the OLED glass substrate studied by the ray-tracing method. As shown, the



FIG. 1. A cross-section of the OLED studied by the FDTD method.
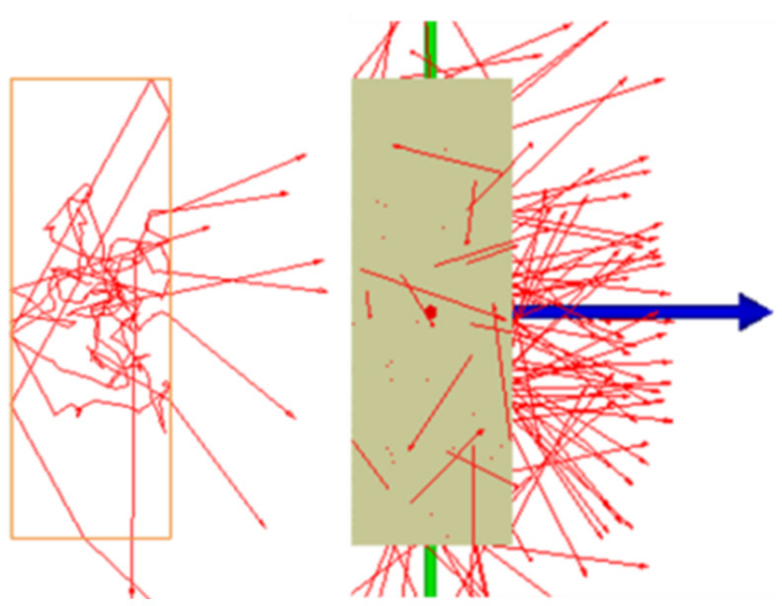

FIG. 2. A schematic figure of the glass substrate in which scattering particles are embedded. The red arrows indicate optical rays. rays are randomly scattered and change directions abruptly in the glass substrate. The particles incorporated into the glass substrate behaved as Mie scatterers. Fig. 3 shows the scattering intensity of the incident light as a function of the scattering angle at a few particle radii. If the particle radius approaches the wavelength of the visible light, the distribution of the forward scattering becomes broad and small oscillatory peaks are observed at high angles, which can be obtained from classical electromagnetic theory.

Pillow-shaped lenses were placed on the front surface of the glass substrate to further increase the OCE. The surface was modified to increase the critical angle and thus, decrease the loss of light by total internal reflection. The radius was optimized, and the configuration of pillow lenses on the glass substrate was varied among $1 \times 1,3 \times 3$ and $5 \times 5$ arrays. Fig. 4 shows the case of a $3 \times 3$ array of pillow lenses.

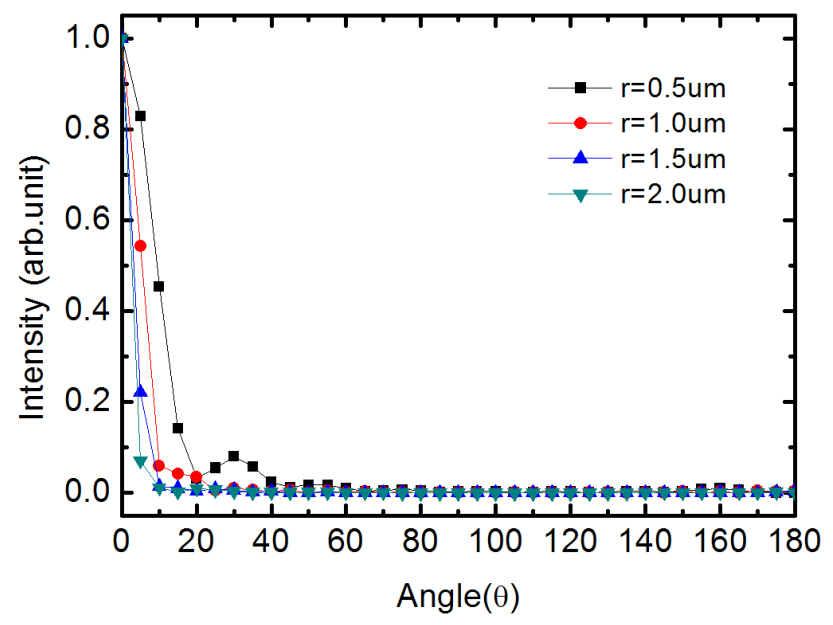

FIG. 3. The angular distribution of the scattering intensity as functions of the angle and the radius of the scattering particles.

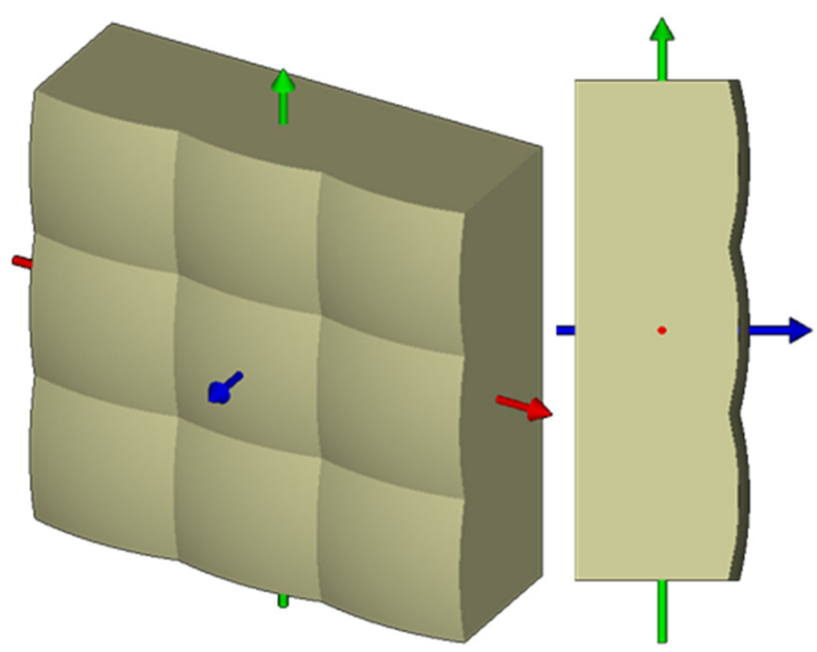

FIG. 4. Pillow Lenses formed on the front surface of the glass substrate. 


\section{RESULTS AND DISCUSSION}

Figure 5 (a) and (b) show a polar plot of the far-field intensity distribution proportional to $|E|^{2}$, where $E$ is the electric field, for the two cases in which the thickness of the ETL $\left(t_{\mathrm{ETL}}\right)$ was $80 \mathrm{~nm}$ and $170 \mathrm{~nm}$, respectively. The on-axis intensity for $t_{\mathrm{ETL}}=80 \mathrm{~nm}$ is much stronger than that for $t_{\mathrm{ETL}}=170 \mathrm{~nm}$; this difference was attributed to the change in the number of waveguide modes, which is also related to the microcavity effect between the cathode and the ITO [12].

Figure 6 shows the dependence of the OCE on the density and the radius of the Mie scatterers embedded in the glass substrate. This result clearly shows that the OCE displays a maximum at a certain particle density of $10^{7} \sim 10^{8} \mathrm{~mm}^{-3}$ depending on the particle radius. If the density is too low, the scattering effect is not sufficient to disturb the rays propagating through the glass substrate, resulting in almost no increase in the outcoupling efficiency. Conversely, if the density is too high, light cannot propagate easily due to excessively large scattering events, resulting in a decrease in the efficiency. Similar results were reported in Ref. [13]

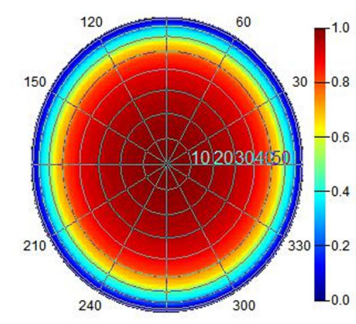

(a)

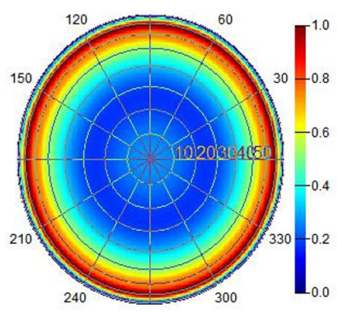

(b)
FIG. 5. A polar plot of the far-field intensity distribution proportional to $|E|^{2}$, where $E$ is the electric field, for the two cases in which the thickness of the ETL ( $\left.t_{\mathrm{ETL}}\right)$ was (a) $80 \mathrm{~nm}$ and (b) $170 \mathrm{~nm}$.

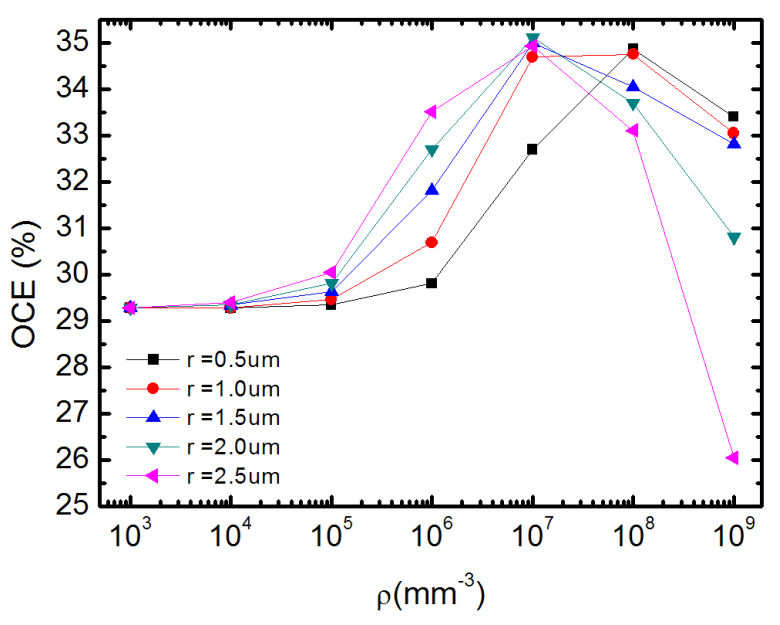

FIG. 6. The dependence of the outcoupling efficiency on the density and the radius of the Mie scatterers embedded in the glass substrate at $t_{\mathrm{ETL}}=80 \mathrm{~nm}$. for a scattering layer inserted between a normal glass substrate and an ITO coating. The maximum OCE achieved was approximately $34.9 \sim 35.1 \%$ at a density of $10^{7} \mathrm{~mm}^{-3}$ and a radius of $1.5 \sim 2.5 \mu \mathrm{m}$.

Figure 7 shows the OCE as a function of the radius for

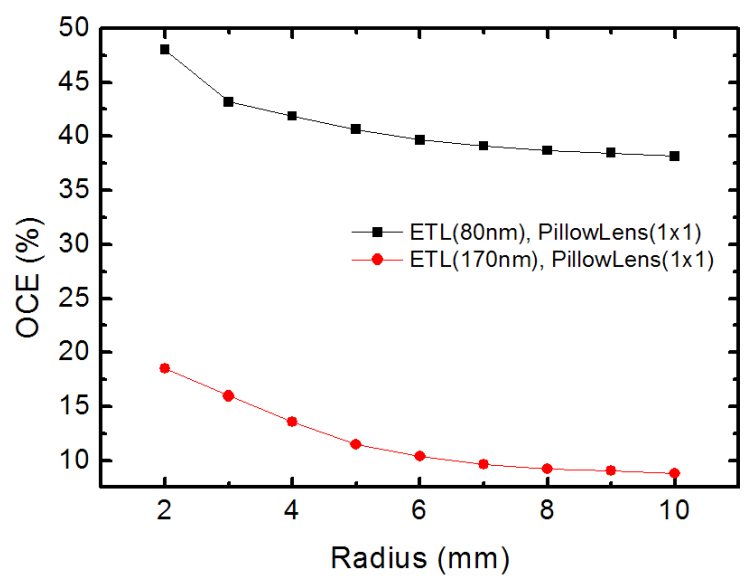

(a)

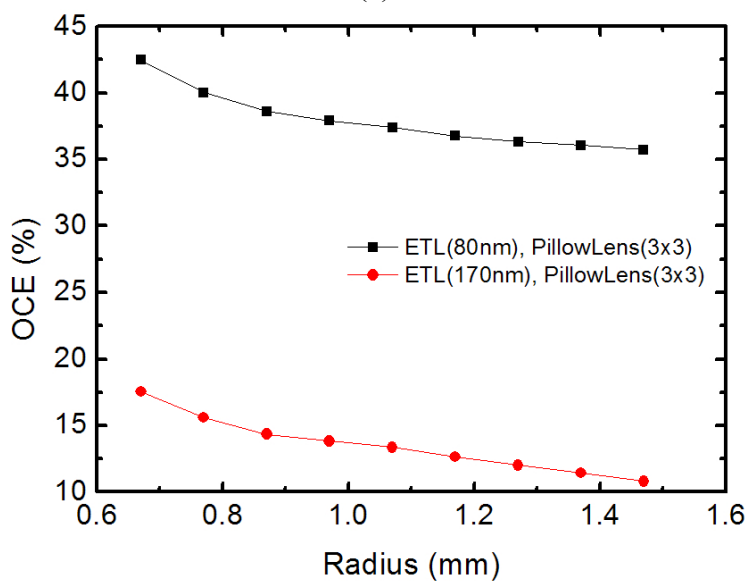

(b)

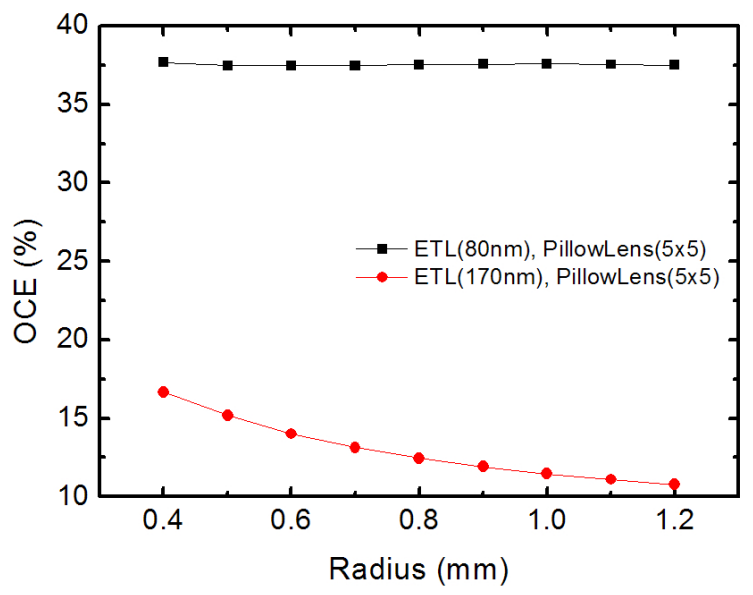

(c)

FIG. 7. OCE as a function of the radius for (a) $1 \times 1$, (b) $3 \times 3$ and (c) $5 \times 5$ pillow lens arrays. The black symbols correspond to the data for $t_{\mathrm{ETL}}=80 \mathrm{~nm}$, while the red symbols to the data for $t_{\mathrm{ETL}}=170 \mathrm{~nm}$. The particle density was fixed at $10^{7} \mathrm{~mm}^{-3}$. 
the three cases, i.e., (a) $1 \times 1$, (b) $3 \times 3$ and (c) $5 \times 5$ pillow lens arrays. It is worth noting that the OCE is very sensitive to the ETL thickness. The placement of pillow lenses on the front surface of the glass substrate increases the OCE from $35 \%$ to a maximum of $\sim 48 \%$ at $t_{\mathrm{ETL}}=80$ nm. In the case of a single pillow lens (Fig. 7(a)), the OCE increases to approximately $48 \%$ at a radius of $2 \mathrm{~mm}$. This behavior occurs because the emitting source is located at the center of the glass substrate. On the other hand, the OCE for the case of $t_{\mathrm{ETL}}=170 \mathrm{~nm}$ increases approximately by a factor of two as the radius of the pillow lens array decreases. This OCE enhancement factor for the case of $t_{\mathrm{ETL}}=170 \mathrm{~nm}$ is larger than that for the case of $t_{\mathrm{ETL}}=80 \mathrm{~nm}$. This result is related to the fact that the intensity distribution in the glass substrate depends on the ETL thickness significantly as shown in Fig. 5. The intensity at $t_{\mathrm{ETL}}=170 \mathrm{~nm}$, shown in Fig. $5(\mathrm{~b})$, is very weak along the normal direction and much of the light energy is emitted toward high angles in the glass substrate, which is due to the destructive interference as described below. The curved surfaces of the pillow lenses are effective in outcoupling these high-angle rays via refraction. Since the relative portion of the high-angle rays among the total amount of light is much larger for the case of $t_{\mathrm{ETL}}=170$ $\mathrm{nm}$, we can expect the OCE will be more enhanced than the case of $t_{\mathrm{ETL}}=80 \mathrm{~nm}$.

Figure 8 shows the dependence of the ratio in each of the three modes (waveguide mode, glass mode, and the air mode) on the thickness of ETL. The waveguide and glass modes are indicative of the light trapped in the organic layer and the glass substrate, respectively. Here, the waveguide mode partly includes cathode loss, sometimes called surface plasmon loss. The air mode represents the amount of light that escapes from the OLED and thus determines the outcoupling efficiency. The ratios of these three modes depend significantly on the ETL thickness and show some oscillation. The air mode exhibits two maxima at approximately 80 and $260 \mathrm{~nm}$ within the examined thickness range. This result is very similar to the results of previous calculations and simulations $[12,14]$. As shown in Fig. 8, the OCE becomes much weaker at $t_{\mathrm{ETL}}=170 \mathrm{~nm}$ than at $t_{\mathrm{ETL}}=80 \mathrm{~nm}$. If we consider the microcavity effect between the cathode and ITO, the difference in the optical path length between the two planar mirrors (i.e., the cathode and the ITO electrode) is $2 n L$, where $n$ and $L$ are the refractive index and the thickness of the ETL layer, respectively. Considering the phase shift of $\pi$ which the light reflected at the surface of the cathode experiences, it is easily calculated that the constructive interference occurs at approximately 80 and $242 \mathrm{~nm}$. These two lengths are consistent with those at which OCE becomes maxima as shown in Fig. 8 and correspond to the fundamental and first excited modes [15]. Therefore, the low OCE at $t_{\mathrm{ETL}}=170 \mathrm{~nm}$, shown in Fig. 8(a), is attributed to the destructive interference in the microcavity.

The maximum value of the OCE can be increased by incorporating scattering particles into the glass substrate. Fig. 8 (b) shows that the maximum OCE at approximately $80 \mathrm{~nm}$ increases from $29.0 \%$ to $35.1 \%$ when the density of the scattering particle at the radius of $2.0 \mu \mathrm{m}$ is $10^{7} \mathrm{~mm}^{-3}$. If one pillow lens with the radius of $2 \mathrm{~mm}$ is added to the substrate without scattering particles, the maximum OCE increases to $48.0 \%$. However, the combination of pillow lenses and scattering particles results in a minute increase in the OCE of $0.4 \%$, indicating that the pillow lens structure is much more effective in outcoupling the light guided in OLED into the air. Finally, we should note that the change in the thicknesses of the HTL and ITO also results in similar oscillatory behavior for the OCE because these layers control the allowed number of modes in the waveguide, which consisted of an ETL, an HTL and ITO [12]. However, the most drastic change is usually observed after modifying the ETL thickness because the distance between the location of the radiating dipole and the cathode, which is determined

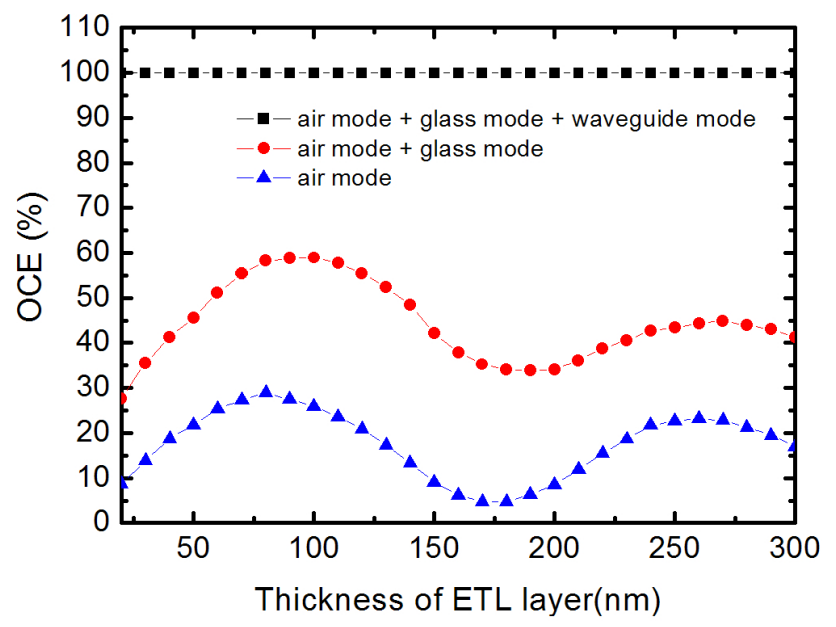

(a)

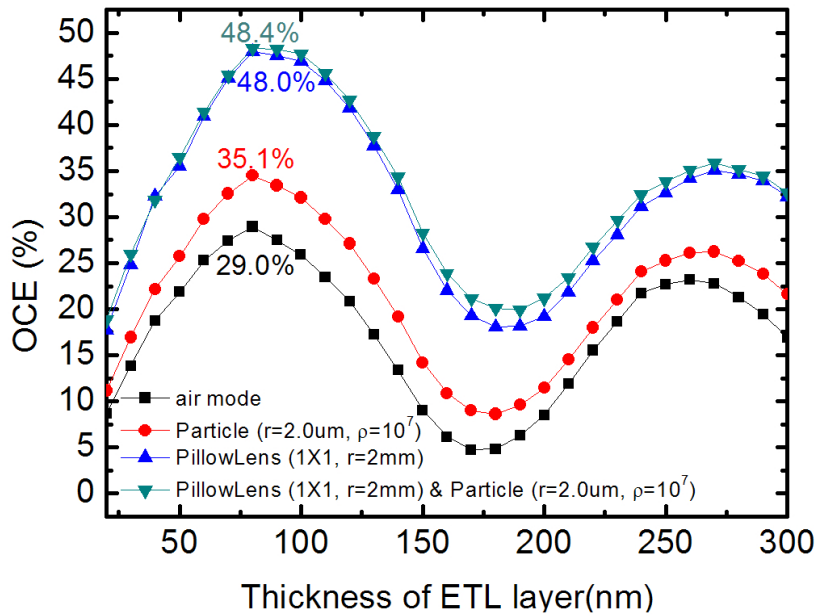

(b)

FIG. 8. (a) The dependence of the ratio in each of the three modes as a function of the ETL thickness without scattering particles and pillow lenses. (b) The dependence of the air mode on the ETL thickness obtained under various conditions. 
by the ETL thickness is the most important parameter affecting the microcavity effect of OLEDs [16].

The increase in the OCE by adopting scattering particles in the substrate was experimentally demonstrated by previous studies [13, 17-18]. Barthelt et al., reported that the application of scattering films on the glass substrate could increase the OCE by approximately $22 \%$ [18], which is close to the value obtained by this study. In some studies $[13,17]$, the refractive index of the substrate was controlled to be close to that of ITO/organic layers. In this case, the trapped waveguide mode can also be extracted via scattering resulting in much higher OCE, because there is nearly no index barrier between the substrate and the ITO/organic layers. On the other hand, there have been many studies on the effect of lenses on the OCE [19-21]. Application of a hemi-spherical lens on the substrate was found to increase the OCE by 1.6 3.0 depending on the refractive index and lens parameters [19]. Using a single lens on OLEDs, however, increases the thickness of the OLED device. In this context, microlens-based optical structures are preferred for practical applications [20-21]. Compared to the microlenses of which the cross-section is circular on the substrate, the fill factor of pillow lenses, which is $100 \%$, is much higher. Therefore, it would be interesting to compare systematically the optical performances of various microlenses with different fill factors. In addition, the intensity distribution on OLED is expected to depend on the detailed shape of microlenses and other optical structures adopted for enhancing OCE. The far-field intensity distribution depending on the scattering particles and pillow lenses was investigated and will be reported elsewhere [22].

Finally, we would like to point out that an isotropic point dipole was assumed for the simulation. However, it is now widely recognized that the emitter orientation is closely related to the coupling between the surface plasmons and the radiating dipole and thus has substantial effect on the device efficiency [2]. In this respect, polarization-dependent optical modeling and analysis [23] are important for getting more insights into the optical power distribution in OLEDs, which is under progress and will be reported in the forthcoming article.

\section{CONCLUSION}

The effect of the density and the radius of Mie scatterers embedded in a glass substrate on the outcoupling efficiency of an OLED was studied using optical simulation. The finite-difference time-domain method was used to obtain the intensity distribution on the transparent electrode of the OLED. This distribution was in turn used as a light source to carry out a ray-tracing simulation of the OLED with a diffusing substrate. The OCE was found to be very sensitive to the thickness of the ETL, which was related to the change in the number of allowed waveguide modes in the organic layer. The incorporation of Mie scatters with a radius of
$2.0 \mu \mathrm{m}$ into the glass substrate at the density of $10^{7} \mathrm{~mm}^{-3}$ increased the OCE by $21.0 \%$. Application of one pillow lens with a radius of $2 \mathrm{~mm}$ on the front surface of the glass substrate increased the OCE by $65.5 \%$. The present simulation study revealed that the OCE of bottom-emitting OLEDs could be enhanced by adopting scattering substrate or pillow lenses formed on the substrate, which should be combined by the optimization of the thicknesses of each layer in OLED, in particular, the electron-transporting layer.

\section{ACKNOWLEDGMENT}

This work was supported by the Hallym University Research Fund, 2012 (HRF-G-2012-3).

\section{REFERENCES}

1. K. Hong and J.-L. Lee, "Recent developments in light extraction technologies of organic light emitting diodes," Electron. Mater. Lett. 7, 77-91 (2011).

2. W. Brütting, J. Frischeisen, T. D. Schmidt, B. J. Scholz, and C. Mayr, "Device efficiency of organic light-emitting diodes: progress by improved light outcoupling," Phys. Status Solidi A 210, 44-65 (2012).

3. G. Gu, D. Z. Garbuzov, P. E. Burrows, S. Venkatesh, and S. R. Forrest, "High-external-quantum-efficiency organic light-emitting devices," Opt. Lett. 22, 396-398 (1997).

4. J.-H. Jang, M.-C. Oh, T.-H. Yoon, and J. C. Kim, "Polymer grating imbedded organic light emitting diodes with improved out-coupling efficiency," Appl. Phys. Lett. 97, 123302 (2010).

5. C. S. Choi, S.-M. Lee, M. S. Lim, K. C. Choi, D. Kim, D. Y. Jeon, Y. Yang, and O. O. Park, "Improved light extraction efficiency in organic light emitting diodes with a perforated $\mathrm{WO}_{3}$ hole injection layer fabricated by use of colloidal lithography," Opt. Express 20, A309-A318 (2012).

6. Y.-H. Cheng, J.-L. Wu, C.-H. Cheng, K.-C. Syao, and M.-C. M. Lee, "Enhanced light outcoupling in a thin film by texturing meshed surfaces," Appl. Phys. Lett. 90, 091102 (2007).

7. C.-C. Liu, S.-H. Liu, K.-C. Tien, M.-H. Hsu, H.-W. Chang, C.-K. Chang, C.-J. Yang, and C.-C. Wu, "Microcavity top-emitting organic light-emitting devices integrated with diffusers for simultaneous enhancement of efficiencies and viewing characteristics," Appl. Phys. Lett. 94, 103302 (2009).

8. C.-H. Chang, K.-Y. Chang, Y.-J. Lo, S.-J. Chang, and H.-H. Chang, "Fourfold power efficiency improvement in organic light-emitting devices using an embedded nanocomposite scattering layer," Org. Electron. 13, 1073-1080 (2012).

9. S. W. Liu, J. X. Wang, Y. Divayana, K. Dev, S. T. Tan, H. V. Demir, and X. W. Sun, "An efficient non-Lambertian organic light-emitting diode using imprinted submicron-size zinc oxide pillar arrays," Appl. Phys. Lett. 102, 053305 (2013).

10. S. S. Jeong and J.-H. Ko, "Simulation study on the optical structures for improving outcoupling efficiency of organic light emitting diodes,” J. Inf. Disp. 13, 139-143 (2012). 
11. Y.-J. Lee, S.-H. Kim, G.-H. Kim, and Y.-H. Lee, "Far-field radiation of photonic crystal organic light-emitting diode," Opt. Express 13, 5864-5870 (2005).

12. A. Chutinan, K. Ishihara, T. Asano, M. Fujita, and S. Noda, "Theoretical analysis on light-extraction efficiency of organic light-emitting diodes using FDTD and mode-expansion methods," Org. Electron. 6, 3-9 (2005).

13. N. Nakamura, N. Fukumoto, F. Sinapi, N. Wada, Y. Aoki, and K. Maeda, "Glass substrates for OLED lighting with high out-coupling efficiency," in Proc. SID'09 Technical Digest (San Antonio, USA, 2009), pp. 603-607.

14. R. Meerheim, M. Furno, S. Hofmann, B. Lussem, and K. Leo, "Quantification of energy loss mechanisms in organic light-emitting diodes,” Appl. Phys. Lett. 97, 253305 (2010).

15. E. F. Schubert, N. E. J. Hunt, R. J. Malik, M. Micovic, and D. L. Miller, "Temperature and modulation characteristics of resonant-cavity light-emitting diodes," J. Lightwave Technol. 14, 1721-1729 (1996).

16. D. G. Deppe, C. Lei, C. C. Lin, and L. Huffaker, "Spontaneous emission from planar microstructures," J. Modern Optics 41, 325-344 (1994).

17. T. Nakamura, H. Fujii, N. Juni, and N. Tsutsumi, "Enhanced coupling of light from organic electroluminescent device using diffusive particle dispersed high refractive index resin substrate," Opt. Rev. 13, 104-110 (2006).
18. R. Bathelt, D. Buchhauser, C. Gärditz, R. Paetzold, and P. Wellmann, "Light extraction from OLEDs for lighting applications through light scattering," Org. Electron. 8, 293-299 (2007).

19. C. F. Madigan, M.-H. Lu, and J. C. Sturm, "Improvement of output coupling efficiency of organic light-emitting diodes by backside substrate modification," Appl. Phys. Lett. 76, 1650-1652 (2000).

20. Y. Sun and S. R. Forrest, "Organic light emitting devices with enhanced outcoupling via microlenses fabricated by imprint lithography," J. Appl. Phys. 100, 073106 (2006).

21. B. C. Krummacher, M. K. Mathai, V. Choong, S. A. Choulis, F. So, and A. Winnacker, "General method to evaluate substrate surface modification techniques for light extraction enhancement of organic light emitting diodes," J. Appl. Phys. 100, 054702 (2006).

22. S. S. Jeong and J.-H. Ko, "Far-field luminous intensity distribution depending on the outcoupling structure of organic light-emitting diodes," J. Inf. Disp., http://www.tandfonline.com/ doi/full/10.1080/15980316.2013.801927.

23. J. Kim, S. Jung, and I. Jeong, "Optical modeling for polarization-dependent optical power dissipation of thin-film organic solar cells at oblique incidence," J. Opt. Soc. Korea 16, 6-12 (2012). 